\title{
A biopsychosocial approach in physical therapy to treat a patient with chronic osteoarthritic associated knee pain
}

\author{
Tye Yetzer, SPT and Heather Disney, PT, DPT, MTC, OCS* \\ University of Saint Augustine for Health Sciences, San Marcos Campus, USA
}

\begin{abstract}
Background and purpose: Chronic pain can have a significant impact on patients in the realm of orthopedic physical therapy. These patients can develop underlying biopsychosocial issues and behaviors that can significantly affect their progress with physical therapy. The purpose of this report was to examine the role of pain behavior and patient education on functional mobility in a patient with chronic osteoarthritic associated knee pain.

Case description: A 53-year-old female with history of chronic pain presents to physical therapy with an exacerbation of right knee pain following a mechanical fall at work.

Intervention: Patient education regarding the degenerative nature of the condition and avoidance of painful behaviors. Other interventions included joint mobilization, strengthening exercises, interferential current and icing as a modality were included.

Outcomes: The patient did not improve per objective measures used to measure range of motion, pain, joint mobility, strength, and the Lower Extremity Functional Scale. However, the patient expressed improvement in standing and gait tolerance and her ability to complete vocational tasks at work.

Discussion: Earlier recognition of biopsychosocial factors and the proper referral to the correct medical professionals may have helped this patient increase progress and achieve goals set with physical therapy sooner. With the number of patients that suffer from chronic pain, it may be necessary to include further education on chronic pain behaviors, their implications, and how to recognize them early in the rehabilitation process in the student physical therapist curriculum.
\end{abstract}

\section{Introduction}

Chronic pain is a significant issue affecting many individuals across the country. Research has shown that as many as 100 million Americans, nearly $1 / 3$ of the population, suffer from some type of chronic pain [1]. When individuals suffer from pain for long periods of time they may develop new behaviors in response to that pain for a variety of reasons. These behaviors may be a major contributing factor to the patient's progress with physical therapy treatment. A key premise for satisfactory treatment is awareness of the student physical therapist to the multiple factors influencing symptoms and functional limitations of patients with chronic pain [2]. A comprehensive assessment is needed that addresses musculoskeletal, psychosocial, and behavioral domains as they contribute to chronic pain, decreased functional mobility, and presentation in the clinic [2].

Chronic pain is described as a persistent pain that is not amenable to treatments based on specific remedies or to routine methods of pain control and does not serve as a biologic purpose indicative of tissue damage or irritation [3]. In subjects with chronic pain, the body is unable to restore its physiological functions to normal homeostatic levels and will often produce significant changes in mood and lifestyle [3]. Changes in behavior developed from chronic pain can manifest in many ways. Examples include malingering or false insurance claims for monetary gain, becoming accustomed to sympathy and attention from others, avoidance of undesirable vocational tasks and duties, and changes in functional movement patterns. When chronic pain is not relieved and learned pain behaviors are reinforced, these can produce declines in function and lead to decreased participation in a person's normal activities and required vocational tasks [3].
Evidence also suggests that pain disability is not only a factor of the intensity of pain, but also by the patient's interpretation of that pain $[4,5]$. Thus, educating patient on the cause and prognosis of their pain and retraining them to minimize and eliminate learned compensations may be beneficial to their functional and vocational task performance. A study of military soldiers suffering from low back pain found that brief psychosocial education consisting of evidenced based information designed to reduce fear of low back pain and encourage active coping strategies was more beneficial than exercise alone in preventing future medical visits [6]. Education may play an important role in helping decrease our patients pain and achieving our goals in the clinic.

A newer theme in physical therapy is the idea of patientcenteredness. In the acute hospital setting there has been a large movement to incorporate patient first care. A recent literature review by Wijma, et al. cited eight characteristics of patient-centered care: respect for patient values and needs, coordinated and integrated care, high quality information and education for the patient and family, physical comfort, emotional support, involvement of family, continuity of care, and access to required care [7]. Further research has shown that utilizing the patient-centered approach can increase

Correspondence to: Heather Disney, PT, DPT, MTC, OCS, University of Saint Augustine for Health Sciences, San Marcos Campus, USA, Tel: 6192064969; E-mail: hdisney@usa.edu

Key words: biopsychosocial, physical therapy, osteoarthritis, knee pain, manual therapy

Received: November 11, 2017; Accepted: November 27, 2017; Published: November 30, 2017 
patient education, clarify the patient's concerns and beliefs, and have a positive effect on objective measures [8]. During their literature review they found that there is a lack of understanding surrounding the idea of patient-centered care in physical therapy. This may have large effect on our outcomes in the clinic since our profession relies heavily on patient autonomy and self-management. If physical therapists are able to treat the patient while incorporating these characteristics of patientcenteredness they may have more success in getting the patient to maximally participate in the plan of care.

In this case report, the patient did not show much improvement through objective measures she was able to improve her tolerance and activity performance during vocational tasks. The purpose of this report is to examine the role of pain behavior and patient education on functional mobility in a patient with chronic osteoarthritic associated knee pain.

\section{Case description}

The subject was a 53-year-old Caucasian female histology department supervisor at a level 3 medical center who was diagnosed with right sided osteoarthritic associated knee pain following a fall at work. She was 5'5" tall, weighing 176 pounds, giving her an overweight BMI of 29.3. She reported that she had stood and was taking her first step when she caught her right foot on the corner of a desk and fell directly onto the right knee, elbow, and shoulder. The patient reported she did not visit her MD for two weeks following the fall and that her pain had slightly improved during those two weeks. At that visit, the MD had referred her to physical therapy and it was an additional two weeks before her initial evaluation in the PT clinic. During this one month period post falling she was able to complete her work day with modifications in order to decrease the amount of time spent on her feet, such as frequent seated rest breaks and combining multiple tasks into single trips through the lab and to other floors.

She presented to the clinic with a 4-week history of knee pain and joint tenderness, mostly with weight bearing activity. Symptoms were provoked with ambulation, prolonged periods of standing, and while completing work tasks like moving between work stations and ascending stairs. At the time of the evaluation the patient was limited to standing for less than 5 minutes and could only perform vocational tasks for less than 30 minutes at a time due to $8 / 10$ knee pain described as aching, sore, and sometimes shooting. Pain would subside to 4-5/10 within 15 minutes once resting and non-weight bearing to the left lower extremity. She has a long-standing history of knee pain since 2012 at which time she was diagnosed with osteoarthritis in her right knee. Since 2012 she has managed her knee pain well and medical records indicated she had undergone successful conventional treatment including physical therapy for osteoarthritic left knee pain in 2015. While this patient was seen for right knee pain she was also being treated by physical therapy for cervical spine pain and by occupational therapy for right elbow lateral and medical epicondylitis and right upper extremity myositis.

Past medical history included right knee osteoarthritis stage 2, osteoporosis diagnosed in 2013, systemic lupus erythematosus (SLE) diagnosed during childhood, hypothyroidism diagnosed in 2009 , and hypertension diagnosed in 2007. Current medications include levoxyl for hypothyroidism, doxepin for depression, benlysta for SLE, and naproxen for pain and inflammation. The patient is a single parent who has an adult child in his early 20's with a developmental disorder limiting her availability to only once a week for therapy. Her son works a local retail job, but is unable to operate a motor vehicle due to a life long history of seizures, therefore she provides daily transportation for him. The patient was encouraged to participate in therapy more than one day a week, especially since the one day she was currently scheduled for therapy she was being seen for her knee, neck, and elbow in succession. Though maximally encouraged, the patient was adamant that coming to the clinic more than one day a week was not an option at that time.

\section{Patient evaluation}

After completing a thorough lower extremity evaluation, multiple impairments were found that were associated with the function of the affected extremity. Primary impairments include right knee pain with ambulation, decreased right knee AROM in both extension and flexion, [9] decreased PROM accessory at the left tibiofemoral joint in a $\mathrm{P} / \mathrm{A}$ and $\mathrm{A} / \mathrm{P}$ direction with tight capsular end feel, inability to grade quadriceps strength due to pain, and decreased left hamstrings and glute maximus strength [10]. She presented with slightly increased kyphosis and mild forward head posture that was being addressed during her cervical spine treatment. During gait patient exhibits left lower extremity decreased stance time and mild genu valgus when compared to right knee. Activities this patient has difficulty with ability to bear weight through left lower extremity and ambulating more than 5 mins. This is affecting her participation negatively at work in which she is on her feet for $60 \%$ of her day standing at a workstation and moving between different areas of the lab. She works at a large medical center and often has to move samples and supplies between different floors which she is able to complete with significant pain that worsens as the time on her feet during the day increases. She has modified some of her work activities and has had to increase the number of seated rest breaks throughout her day, but much of her job requires being on her feet moving between the different work stations. She also expresses difficulty with tasks at home like cleaning and shopping, stating she had been putting some tasks off because of the high level of pain in the knee at the end of her work day. The physical therapy diagnosis was ICD10-CM code 26.2: Difficulty in walking [11]. Her medical diagnosis upon referral was M17.9: Osteoarthritis of the knee, unspecified. The practice pattern was 4E "Impaired Joint Mobility, Motor Function, Muscle Performance, and Range of Motion Associated with Localized Inflammation" [12]. These findings were used to create a safe plan of care.

\section{Plan of care}

Interventional goals were created based on the patients' evaluation and overall functional mobility. One primary goal was to return active knee range of motion to zero degrees extension to improve gait quality, standing balance, and functional mobility. The passive range of motion accessory goal was to normalize anterior and posterior glides at the tibiofemoral joint to $3 / 6$ grades to improve joint kinematics and improve functional mobility. The strength goal focused on increasing knee flexion, extension, and hip external rotator groups to at least a $4+/ 5$ strength to normalize gait and decrease the genu valgus seen during the movement analysis. The patient had decreased gait tolerance due to pain indicating a goal to increase her ambulation time to 5 minutes with less than $2 / 10$ pain in order to complete her required vocational tasks. The outcome goal at the time of discharge was to increase the patient's LEFS score to $80 / 80$ indicating no difficulty with lower extremity function.

The patient was middle aged, non-active, and had an extensive past medical history with multiple comorbidities who suffered an acute dysfunction of her tibiofemoral joint. The patient's prognosis was fair based on her sedentary lifestyle, comorbidities, and the degenerative nature of osteoarthritis. 
Intervention plan included strengthening, range of motion exercise, joint manipulation, modalities, and functional training. Treatment strategy focused on therapeutic exercise to increase lower extremity strength and function, primarily of the gluteus maximus and gluteus medius. Joint manipulation was used for pain relief and to improve mobility of the joint capsule to allow for normal arthrokinematics during ambulation. Functional training to prevent medial collapse at the tibio-femoral joint was also a focus to facilitate a pain free gait pattern. Modalities including electrical stimulation and hot/cold therapy were utilized as needed to provide pain relief.

The time required to reach all goals was expected be 16 visits, occurring once a week for 16 weeks. The patient had concerns with committing to a frequency of twice a week because she was a single parent and had a young adult son with a learning disability who she provided transportation and other necessities for. For that reason, the patient attended physical therapy once a week as agreed upon with the student physical therapist, after educating the patient on the importance of completing a home exercise plan (HEP) in order to reach our outcome goals. The patient was educated and provided a HEP throughout the duration of treatment starting with range of motion exercises on the initial evaluation.

\section{Implementation of intervention/Re-assessment}

The patient was instructed to perform her HEP 5 days of the week and not on days when she had a physical therapy session in the clinic. She was educated on remaining in pain free motion, importance of breathing throughout exercise, and slow, controlled movement. If significant pain was experienced with any exercise, she was instructed to terminate that exercise and then to provide that information to the therapist at the next session so that it could be addressed. The chart below in Table 1shows an outline of the specific exercises utilized in the HEP during the treatment (Table 1).

Resistance exercise also occurred within the clinic with a primary focus on the abductors and external rotators of the hip with intent to help decrease the genu valgus seen during functional mobility by providing better control of the knee through the hip and ankle. Decreased hip abductor and external rotator strength has been shown to result in an increased knee valgus [13] by allowing internal rotation of the femur which results in medial collapse at the knee joint, causing distraction with the medial compart of the knee and increased compression of the lateral compartment. It was felt this was the major contributing factor to her lateral knee pain.

Grade I/II joint manipulation was utilized for palliative effects and to provide some relief prior to other interventions in the clinic. Grade III/IV joint manipulation was used to increase mobility of the joint in aim of increasing the space between joint surfaces. For all manipulations, the patient was supine and supported for comfort. Tibiofemoral joint was supported in the loose pack position of 10-20 degrees of flexion [14], joint alignment was assessed, and the patient was instructed to breath and remain relaxed. To provide pain relief the long axis distraction technique (LAD) was used with grade I/II movements in order to distract the articular surfaces and prevent their irritation [14,15]. Grade III/IV anterior and posterior glides of the tibia on the femur were used along with the LAD to improve the mobility of the anterior and posterior capsule and facilitate the restoration of those specific component motions to achieve our goal of full knee range of motion $[14,15]$. The patient was able to tolerate manipulation well with occasional soreness with hand pressure to the proximal tibia rated 5/10 pain. With an adjustment of hand position treatment was continued.
During the initial duration of treatment the patient responded well to the LAD technique in terms of pain, often expressing a decrease in her numerical pain score of up to two points post treatment [16], when combined with a cold pack to the knee for 15 minutes.

One observation made during the movement analysis in the initial evaluation was that with gait and stair execution the patient had the most pain when the tibiofemoral joint was at its most valgus position and the pain was located to the lateral aspect of the joint. Studies have shown that women with greater knee valgus may experience biomechanics that potentially increase the load at the lateral tibiofemoral joint [14] and it was determined that this was a contributing factor to pain during these functional movements. These interventions included activities like partial squats, partial lunges, and stair stepping in front of a mirror. The mirror was a tool to provide visual feedback of the patient's body in space and improve her awareness of the affected knee during functional activities [6]. She was instructed to perform the activity while maintaining her knee in alignment over her foot. If pain increased or she was unable to maintain the knee position she was instructed to not continue as far into the specific movement and only perform them until that point. The patient was able to tolerate these functional activities well when she stayed within her pain free range.

Each treatment session was concluded with the use of IFC and ice therapy to decrease any inflammation and reduce pain from activity post treatment [12,17]. A variety of objective measures were used to determine progress toward her functional goals. These objective measures included range of motion [18], manual muscle tests [18], and completion of the lower extremity functional scale [19]. Measurements were taken during the initial evaluation, for re-assessment at the fourth week, and at anticipated discharge in week eight

The patient reported consistent compliance with her HEP throughout the duration of treatment. She did complain of pain with resisted knee extension beginning in week 5 and was advised to only perform that exercise in a pain free motion. Exercise progressions were based on the patient's tolerance, current pain level, and perceived difficulty with certain exercises or activities. During functional movement training the patient was able to achieve $50 \%$ of a full squat in week 3 and $50 \%$ of a full lunge in week 4 . At that point when the patient attempted a deeper position she would complain of increased pain after 1 set of 10 and was then advised to stay in the pain free $50 \%$ of full motion range

\section{Outcomes}

In the eighth week the patient had slightly improved scores in most objective measures and expressed improvements in functional mobility and vocational task completion, but was continuing to deal with the same high pain levels at the end of her workdays. She demonstrated improvements in AROM and strength. Most significantly, she did report increased tolerance to standing and an increase in time during her workday to when the pain would begin, however she felt her pain at the end of the day and overall was the same. This was reflected in her LEFS score at 8 weeks of 46 , which was only 7 points higher than original score and did not meet the minimal detectable change value of 9 for lower extremity osteoarthritis $[19,20]$. We cannot say that the patient's lower extremity function improved per the LEFS, but she did express that she felt physical therapy had helped and she was able to work longer into her day before her knee became significantly painful (Table 2).

In week four, further imaging was recommended to the referring physician due to the patient's lack of progress with physical therapy and 
Table 1. Outline of the specific exercises utilized in the HEP during the treatment

\begin{tabular}{|l|l|l|l|}
\hline Therex & Week $\mathbf{1}$ & Week 3 \\
\hline Isometric Hip Abduction & 10 reps W/5 sec hold & 10 reps W/10 sec hold \\
\hline $\begin{array}{l}\text { Isometric } \\
\text { Hip Extension }\end{array}$ & 10 reps W/5 sec hold & 10 reps W/10 sec hold \\
\hline $\begin{array}{l}\text { AROM } \\
\text { Knee Ext }\end{array}$ & 10 reps W/5 sec hold & 10 reps W/5 sec hold W/Yellow TB \\
\hline $\begin{array}{l}\text { AROM } \\
\text { Knee Flex }\end{array}$ & 10 reps W/5 sec hold & 10 reps W/5 sec hold W/Yellow TB & 10 reps W/15 sec hold \\
\hline A/P Lunges & None & None & $25 \%$ of full lunge X 10 reps \\
\hline Lateral Lunges & None $/ 15$ sec hold & 10 reps W/10 sec hold W/Yellow TB \\
\hline AROM Hip Abduction & None & 10 reps each direction & $50 \%$ of full lunge X 15 reps \\
\hline AROM Hip Extension & None & 10 reps W/Yellow TB TB \\
\hline AROM Hip External rotation & & 10 reps W/Yellow TB \\
\hline
\end{tabular}

Table 2. Patient outcomes

\begin{tabular}{|c|c|c|c|}
\hline & Initial Evaluation & Week 4 & Week 8 \\
\hline $\begin{array}{l}\text { AROM } \\
\text { Flexion } \\
\text { Extension }\end{array}$ & $\begin{array}{l}105 \text { degrees } \\
-14 \text { degrees }\end{array}$ & $\begin{array}{l}111 \text { degrees } \\
-8 \text { degrees }\end{array}$ & $\begin{array}{l}112 \text { degrees } \\
-10 \text { degrees }\end{array}$ \\
\hline $\begin{array}{l}\text { MMT } \\
\text { Quadriceps } \\
\text { Glute Max } \\
\text { Hamstrings }\end{array}$ & $\begin{array}{l}\text { Not maximally tested due to pain } \\
4 / 5 \\
4 / 5\end{array}$ & $\begin{array}{l}\text { Not maximally tested due to pain } \\
4 / 5 \\
4 / 5\end{array}$ & $\begin{array}{l}\text { Not maximally tested due to pain } \\
4+/ 5 \\
4+/ 5\end{array}$ \\
\hline PROM Accessory & $2 / 6$ in both $\mathrm{A} / \mathrm{P} \& \mathrm{P} / \mathrm{A}$ direction & $2 / 6$ in both $\mathrm{A} / \mathrm{P} \& \mathrm{P} / \mathrm{A}$ direction & $2 / 6$ in both $\mathrm{A} / \mathrm{P} \& \mathrm{P} / \mathrm{A}$ direction \\
\hline Standing Tolerance & $<5 \min$ & $10 \mathrm{~min}$ & $15 \mathrm{~min}$ \\
\hline $\begin{array}{l}\text { Vocational Task } \\
\text { Tolerance }\end{array}$ & $<30 \mathrm{~min}$ & 2 hours & 2.5 hours \\
\hline Highest Pain Intensity & $9 / 10$ & $8 / 10$ & $8 / 10$ \\
\hline LEFS & 39 & 50 & 46 \\
\hline
\end{tabular}

her persistent high pain scores. She was not able to get an appointment with her physician till after week eight. During the eighth and final visit, it was recommended the patient continue therapy pending the results of further imaging. Although the patient agreed that improvements in her mobility and vocational task performance had occurred she was concerned about her high levels of pain remaining at the end of her weekday. She opted to hold physical therapy and follow up with her physician for a radiograph of her knee. She did express her motivation to continue with the home exercises that did not cause pain in order to maintain her current level of function no matter what her final decisions were in the medical care of her knee.

The patients level of function upon discharge showed improvements with activity level, but her participation remained the same. She was able to stand, squat, and walk for longer periods before pain became significant and thus was able to continue performing the tasks at work that are necessary. At 8 weeks, she remained not able to walk for enjoyment and participate in most outdoor activities though because of pain.

\section{Discussion}

The most important goal for the patient was being able to increase her activity tolerance in order to complete daily required vocational tasks at her job with minimal pain. Although the patient did not demonstrate statistically significant improvements in the objective measures chosen, she did demonstrate increased activity tolerance with vocational tasks, increased standing tolerance, and verbalized that she felt it had to do with a longer period of time before significant pain set in. The case report was written to demonstrate that by educating the patient on her condition, causes of pain, and painful movement patterns she had developed she gained a better understanding which resulted in improved activity tolerance. It is suggested that although there was no significant evidence of improvement through objective measurements (such as range of motion, manual muscle strength testing, etc.), by incorporating patient education into each treatment, the patient had improved functional outcomes. This furthermore supports the use of patient-centeredness in physical therapy.

One of the functional impairments noticed during the evaluation was the increased valgus force occurring in the affected knee joint during standing which increased during both gait and stair climbing. It has been shown through research that weak hip musculature, namely the muscles responsible for femoral external rotation (gluteus medius, gluteus maximus, Sartorius), can be a major contributor to femoral internal rotation resulting in a valgus collapse at the knee joint $[21,22]$. This valgus moment has been shown to be associated with a higher incidence of knee pain and pathology [23]. The plan of care focused on increasing strength and activation of these weak hip external rotators in order to prevent excessive internal femoral rotation with adduction and decrease the valgus occurring during functional movement. By week 8 the patient had shown improvement in preventing this excessive valgus moment during gait and stair climbing, had expressed an increase in both standing and walking tolerance, as well as an increase in the time during work before significant pain set in. Since there is no objective evidence of a significant change in hip strength or range of motion (both active and passive) occurring it is likely the patient improved due to education on her condition and avoidance of painful movement patterns she had developed at her knee, rather than weakness at the hip or decreased range at the knee.

While teaching the patient about her osteoarthritis she made it clear that this was the first time she had been educated on her condition and she was very interested in what was occurring in her knee. When asked if she had ever looked up information about the condition on her own time she said she had never really thought about it and figured 
her medical doctor would inform her of anything she needed to know. Taking the time to explain the degenerative nature of osteoarthritis and the biomechanics of valgus force at the knee joint compressing the lateral half of the joint and that compression being a cause of pain really appeared to increase her understanding of her pain and understanding why physical therapy was focusing on preventing the knee from increasing valgus during closed kinetic chain activities like stance phase of gait and stair climbing [24,25]. The patient then did very well during partial squats and lunges with keeping her knee above her foot and preventing valgus from occurring. Over the next few weeks we did not see progress with objective measurements of range of motion, muscle strength or pain intensity, however the patient continually expressed her knee pain at work was decreasing and she felt it was due to her awareness of her knee position throughout the day. This understanding of her condition and realization that she could positively affect her pain by being conscious of her movements throughout her work day seemed to be a major turning point in her effort, compliance, and functional progress.

One limitation of this report is that this is a single case report. An area for improvement of future case studies would be psychosocial implications on the patient's progress toward goals. This patient had multiple factors in her history that would indicate a proper screen for potential psychosocial factors would be appropriate including chronic pain in multiple areas of her body (right knee, right elbow, and both lumbar and cervical spine), history of systemic lupus erythematosus, osteoporosis, and hypothyroidism. She was a divorced, single mother with a young adult son who had a learning disability and suffered from chronic seizures. She worked full time and made it clear she did not have much support to assist with taking care of her son. In retrospect, the student physical therapist treating this patient felt there was significant psychosocial barriers present that played a role in the patient's unchanging numerical pain scores during the duration of treatment. If these were recognized at the time of evaluation and the proper referrals made, this patient may have made more progress towards goals during the eight weeks of treatment.

Another limitation of this case report was the treatment frequency of one visit per week. This was the patient's decision and was due to her need to provide transportation and care to her disabled son as discussed previously. If the patient had been able to attend therapy twice a week, she may have made quicker progress and been able to spend more time working on functional movement patterns and avoiding painful behaviors. Although, she did state she was continually compliant with the home exercise program and the education about correct knee biomechanics that she was encouraged to maintain while outside the clinic, so the increased progress with twice a week visits may have been minimal.

After completing research for this case study, this student physical therapist feels that more research into the effects of these biopsychosocial issues is warranted. Not only to provide the best physical therapy for this population, but to establish protocols for screening patients and catching these factors earlier enough to make justified referrals and eliminate some of these barriers to achieving our best outcomes. This information could be used to add further education in the student Doctor of Physical Therapy curriculum and better prepare new graduates to recognize and properly manage these biopsychosocial issues.

\section{References}

1. Institute of Medicine Report from the Committee on Advancing Pain Research, Care, and Education (2011) Relieving Pain in America, A Blueprint for Transforming Prevention, Care, Education and Research. The National Academies Press.

2. Dansie EJ, Turk DC (2013) Assessment of patients with chronic pain. Br J Anaesth 111: 19-25. [Crossref]
3. Hertling D, Kessler R (2006) Management of Common Musculoskeletal Disorders, 4th Edition, Philadelphia, PA. Lippincott, Williams, and Wilkins.

4. Jensen MP, Moore MR, Bockow TB, Ehde DM, Engel JM (2011) Psychosocial Factors and Adjustment to Chronic Pain in Persons With Physical Disabilities: A Systematic Review. Arch Phys Med Rehabil 92: 146-160. [Crossref]

5. Turk DC, Wilson HD (2010) Fear of Pain as a Prognostic Factor in Chronic Pain Conceptual Models, Assessment, and Treatment Implications. Curr Pain Headache Rep 14: 88-95. [Crossref]

6. George SZ, Childs JD, Teyhen DS, Wu SS, Wright AC, et al. (2011) Brief psychosocia education, not core stabilization, reduced incidence of low back pain: results from the Prevention of Low Back Pain in the Military (POLM) cluster randomized trial. BMC Medicine 9: 128.

7. Wijma A, Bletterman AN, Clark JR, Vervoort SCJM, Beetsma A, et al. (2017) Patientcenteredness in physiotherapy: What does it entail? A systematic review of qualitative studies. Physiother Theory Pract 33: 825-840. [Crossref]

8. Dwamena F, Holmes-Rovner M, Gaulden CM, Jorgenson S, Sadigh G, et al (2012) Interventions for providers to promote a patient-centred approach in clinical consultations. Cochrane Database Systematic Review 12: CD003267. [Crossref]

9. Gogia PP, Braatz JH, Rose SJ, Norton BJ (1987) Reliability and validity of goniometric measurements at the knee. Phys Ther 67: 192-195. [Crossref]

10. Wadsworth CT, Krishnan R, Sear M, Harrold J, Nielsen DH (1987) Intrarater reliability of manual muscle testing and hand-held dynametric muscle testing. Phys Ther 67: 1342-1347. [Crossref]

11. American Physical Therapy Association (2001) Guide to Physical Therapist Practice. Second Edition. APTA. Phys Ther 81: 739-746.

12. Brosseau L (2011) Thermotherapy for treatment of osteoarthritis. Cochrane Database Of Systematic Reviews.

13. Cashman GE (2012) The Effect of Weak Hip Abductors or External Rotators on Knee Valgus Kinematics in Healthy Subjects: A Systematic Review. J Sport Rehabil 21: 273-284. [Crossref]

14. Paris S, Patla C (2005) E1 Seminar Manual: Extremity Evaluation and Manipulation. University of St. Augustine. St. Augustine, Fl.

15. Paris S, Loubert P (1999) Foundations of Clinical Orthopaedics seminar manual University of St. Augustine. St. Augustine, FL.

16. Childs JD, Piva SR, Fritz JM (2005) Responsiveness of the numeric pain rating scale in patients with low back pain. Spine (Phila Pa 1976) 30: 1331-1334. [Crossref]

17. Zeng C, Li H, Yang T, Deng ZH, Yang Y, et al. (2015) Electrical stimulation for pain relief in knee osteoarthritis: systematic review and network meta-analysis. Osteoarthritis Cartilage 23: 189-202.

18. Kendall F, et al. (2005) Muscles: Testing and Function, with Posture and Pain Philadelphia, PA: Lippincott Williams \& Wilkins.

19. Binkley JM, Stratford PW, Lott SA, Riddle DL (1999) The Lower Extremity Functiona Scale (LEFS): scale development, measurement properties, and clinical application. North American Orthopaedic Rehabilitation Research Network. Phys Ther 79: 371383. [Crossref]

20. Kennedy DM, Stratford PW, Riddle DL, Hanna SE, Gollish JD (2008) Assessing recovery and establishing prognosis following total knee arthroplasty. Phys Ther 88 : 22-32. [Crossref]

21. Souza R, Powers C (2009) Differences in hip kinematics, muscle strength, and muscle activation between subjects with and without patellofemoral pain. J Orthop Sports Phys Ther 39: 12-19. [Crossref]

22. Souza R, Draper C, Fredericson M, Powers C. Femur rotation and patellofemoral join kinematics: a weight-bearing magnetic resonance imaging analysis. $J$ Orthop Sports Phys Ther 40: 277-285. [Crossref]

23. Powers C (2010) The influence of abnormal hip mechanics on knee injury: a biomechanical perspective. J Orthop Sports Phys Ther 40: 42-51. [Crossref]

24. Brindle T, Mizelle J, Lebiedowska M, Miller J, Stanhope S (2009) Visual and proprioceptive feedback improves knee joint position sense. Knee Surg Sports Traumatol Arthrosc 17: 40-47. [Crossref]

25. Hoch MC, Weinhandl JT (2017) Effect of valgus knee alignment on gait biomechanics in healthy women. J Electromyogr Kinesiol 35: 17-23. [Crossref]

Copyright: (C2017 Yetzer T. This is an open-access article distributed under the terms of the Creative Commons Attribution License, which permits unrestricted use, distribution, and reproduction in any medium, provided the original author and source are credited. 